\title{
Research on Contour Layout Property
}

\author{
Bing Zhang ${ }^{1,}$ a and Jianxun Chen ${ }^{2, b}$ \\ 'Department of Computer Science and Technology, Wuhan University of Science and Technology, \\ Wuhan, 430065, China \\ a568896684@qq.com, ${ }^{\text {b7 } 7500080 @ q q . c o m ~}$
}

\begin{abstract}
Keywords: Delaunay triangulation; B-Spline curve; Contour tracking; Contour labels
\end{abstract}
\begin{abstract}
A key and difficult problem of contour study is to prevent two adjacent smooth contours intersect in generation process. This paper presents an approach for smooth curve generation based on a variety of algorithms. The technique combines Delaunay triangular mesh generation algorithm, Delaunay triangulation for contour tracing algorithm, B-spline curve smoothing and Contour labeling. Since the adjacent smooth curve in the visual problems will have an impact on intersection of the data layer and research, this paper uses spline $\alpha$-B to generate smooth contours. That is formula combination of polylines and curves with factor $\alpha$, by changing the value of the intersection curve segments infinitely close to the fold line(when tracing the contour line graph is generated can't be intersected), so that the intersection curves disjointed. Then, this paper optimizes the labeled on the curve graph avoid the intensive areas.
\end{abstract}

\section{Introduction}

Smooth contours refers to an existing set of data points (coordinates, attribute values) in the plane coordinate system, through a series of arithmetic processing(along a characteristic set value, recognize the position with the same attribute values of discrete points), drawing a smooth curve, and marked out its property values on a smooth curve. Such as, contours, isotherm, rainfall and other lines and isobars. By viewing or compare adjacent pitch contour can be roughly understand the distribution of values. Since the resulting polyline curve during the transition, there may be problems, the two adjacent smooth curves will intersect or cross due to the B-spline curve does not pass through the control points during the formation of a line chart tracing algorithm to smooth the curve B-spline curve after handling this process. So this paper further study the adjacent two curves intersection issues after generating a smooth curve.

Paper [1] introduces the definition of Delaunay Triangulation. Research on time required and time complexity generated by Delaunay triangulation algorithm, compared to three generating Delaunay triangulation method (Ye algorithm points, point by point insertion algorithm, triangulation growth method), presented the advantages and disadvantages of the three algorithms. It describes the advantages of constructing Delaunay triangulation: Speed of network configuration and accuracy. Paper [2] propose an improved method of isoline Network, and explained why the contour tracking, tracing a brief definition of the contour, how contour tracing. On two cases contours were presented a detailed design idea (Non-closed contour tracing, Closed contour tracing). Paper [3] compares a several common contour tracing algorithms, designs a optimization method to presents better results by viewing software surfer methods. Paper [4] further addressed the issue of smoothing for generated polyline contours. It describes the types of B-spline curve and its main properties, several B-spline curve generation method, the implementation and significance of B-spline curve. Paper [5] marked the contour curve after smoothing, contour labeling definition, contours marked generation and contour labeling algorithms, established a simple model for exploration of geology and oil.

\section{Contour Polyline Generation}

Delaunay Triangulation and Voronoi Polygon. If the set of points on the plane $P=\left\{p_{1}, p_{2}, p_{3}, \ldots, p_{n}\right\}$ $\mathrm{N} \geq 3$, all the points on the plane are not collinear, And there is no point in the fourth on three points 
which formed a triangle circumscribed circle or within the circumscribed circle. $d\left(p_{i}, p_{j}\right)$ represents distance between two adjacent points.

Planar point set Voronoi diagram: Hypothesis $\mathrm{S}\left(\mathrm{x}_{\mathrm{i}}, \mathrm{y}_{\mathrm{i}}\right)(\mathrm{i} \in\{1,2,3, \ldots, \mathrm{n}\}, \mathrm{n}$ is discrete point $)$ is a discrete points, The region with the point where the straight line is divided into $\mathrm{n}$ adjacent polygons and the following conditions are met:

1) Each polygon has only one discrete point;

2) Polygon from point $A$ to any point where the take (x, y), satisfies:

$$
\sqrt{\left(x-x_{i}\right)^{2}+\left(y-y_{i}\right)^{2}} \leq \sqrt{\left(x-x_{j}\right)^{2}+\left(y-y_{j}\right)^{2}} \ldots \ldots(i \neq j)
$$

Where when the point is in the polygon are equal.

Definition 1: Voronoi diagram of the connection formed by triangulation all adjacent Voronoi polygon center called Delaunay triangulation, as shown in Fig. 1, black and thick line shows the Voronoi polygon. Light-colored line represents the Delaunay triangulation.

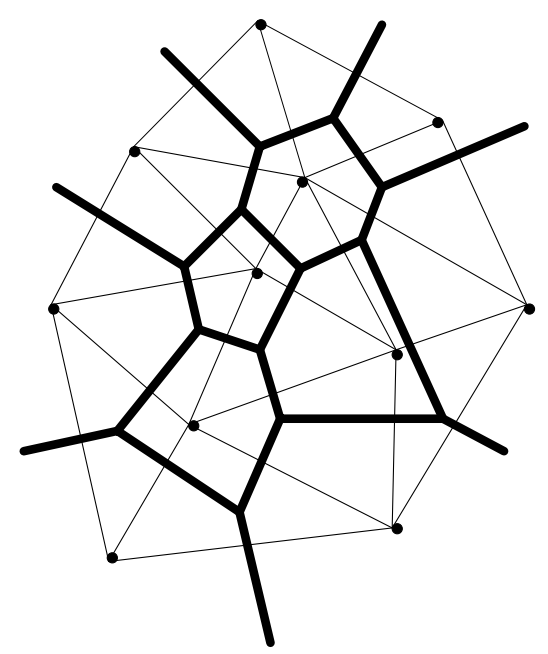

Figure 1. Voronoi diagram

Incremental Insertion Algorithm Delaunay Triangulation Generation. Whether it is with a triangular mesh generation method, incremental insertion points or the rule of law generated by the triangular mesh, it is difficult to meet the features of Delaunay Triangulation. So, presents a LOP optimization method after generating triangulation. Algorithm is as follows:

(1). Find the points with minimum value of $\mathrm{y}, \mathrm{x}$ and maximum value of $\mathrm{y}, \mathrm{x}$ in all discrete points, save the sequence of discrete points. In which two adjacent points pi and pi +1 as a directed line segment pipi+1, from there to the right segment located points, calculate the distance of the line segment to find the farthest point. If there is a point, save the point between pi and pi+1. Repeat the process until each two adjacent points together into a straight line and are not added sequentially decision point is reached. Then, connected all the saved points sequentially to forming a convex polygon that contains all the discrete points.

(2). An initial triangulation generated by connecting any point on convex polygon and convex polygons each vertex.

(3). The remaining points are randomly finding that as pending points. Analyzing the point where the triangle, connecting the point with three vertices of the triangle will generate three new triangles. For each triangle do LOP optimization to meet the third feature of Delaunay triangulation. LOP optimization, as Fig. 2. If the circumcircle of triangle $\mathrm{ABC}$ contains point $\mathrm{D}$, then, swap the diagonal of quadrilateral that formed by point A,B,C and D to generate two new triangles. As Fig. 3. Otherwise, keep the original triangle state as Fig. 4 .

(4). Repeat 3, until all discrete points implement traversing. All discrete point-by-point insertion generates Delaunay triangulation. 


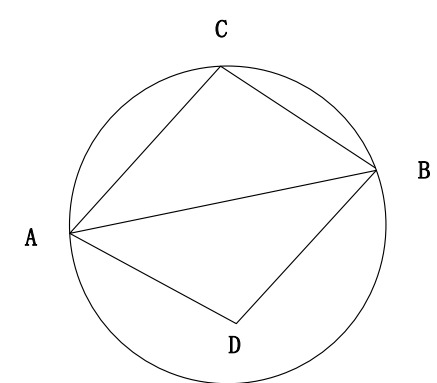

Figure 2

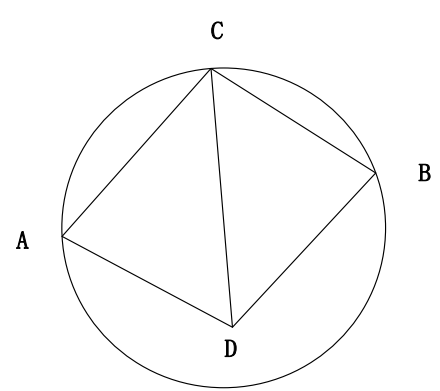

Figure 3

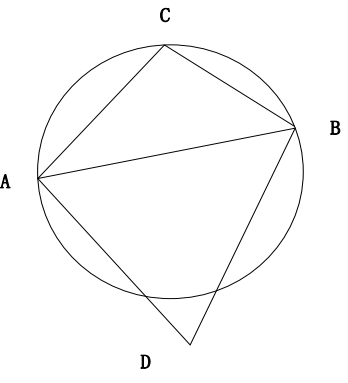

Figure 4

Contour Tracking. Hypothesis three vertices are $\mathrm{P}_{\mathrm{i}}, \mathrm{P}_{\mathrm{i}+1}, \mathrm{P}_{\mathrm{i}+2}$, three vertex coordinates are $\left(\mathrm{x}_{\mathrm{i}}\right.$, $\left.y_{i}, z_{i}\right) 、\left(x_{i+1}, y_{i+1}, z_{i+1}\right) 、\left(x_{i+2}, y_{i+2}, z_{i+2}\right)$ for any triangle, $h$ is the eigenvalue of contours that to be drawn.

The positional relationship between sides of triangle with $\mathrm{h}$ :

$\left(z_{i}-h\right)\left(z_{i+1}-h\right)<0$ contours through edge $P_{i} P_{i+1}$

$\left(z_{i}-h\right)\left(z_{i+1}-h\right)>0$ contours not through edge $P_{i} P_{i+1}$

$\left(z_{i}-h\right)\left(z_{i+1}-h\right)=0$ contours through one vertices or two of the edge $P_{i} P_{i+1}$,

If the contours go through the edge $\mathrm{P}_{\mathrm{i}} \mathrm{P}_{\mathrm{i}+1}$, the contours coordinates $\left(\mathrm{x}_{0}, \mathrm{y}_{0}, \mathrm{z}_{0}\right)$ on edge $\mathrm{P}_{\mathrm{i}} \mathrm{P}_{\mathrm{i}+1}$.can be calculated according to Eq.2.

$$
\left\{\begin{array}{l}
x_{0}=x_{1}+\frac{x_{2}-x_{1}}{z_{2}-z_{1}}\left(h-z_{1}\right) \\
y_{0}=y_{1}+\frac{y_{2}-y_{1}}{z_{2}-z_{1}}\left(h-z_{1}\right) \\
z_{0}=h
\end{array}\right.
$$

\section{Smoothed Contours}

Delaunay triangulation obtained through a series of equivalent points in different areas with Contour Tracing, connected in turn to give a one, however, contour is connected to two adjacent points directly connected to form the line chart. While line charts can also be expressed gentle terrain or changes in vertical distribution of rainfall, but it has affected people's aesthetic effect, so the need for complete tracking of contour maps smoothed.

Common smooth curve algorithm: Linear Iterative Method, B-spline curve, Smooth five-point method and Tension spline interpolation method etc. B-spline curve is smoothed by a series connection consisting of a polynomial curve. B-spline curves are a class of widely used in the CAD (Computer Aided Design) and other computer graphics applications spline. Special cubic B-spline curve is the most common computer uses B-spline curve. This paper uses a cubic B-spline curve.

\section{The Optimization for Layout Attributes of Contour}

The Optimization of B-spline Curve. As a contour generated using the B-spline curves shown in Fig. 5 , since the smooth curve is not through the control points, the distances from the curve to the control points can lead to intersection between two adjacent smooth curves, which is greatly similar to the principle of contour tracing in this paper. Thus, the further improvement for the smooth $\mathrm{B}$-spline curve is made in this part. 


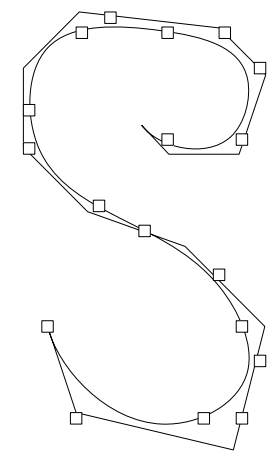

Figure 5. The polygon representing the control points of B-spline curve and Bezier curve

According to Fig. 5 , the intersection problem is not occurred in the control polygon of B-spline based contour tracking while the closer Bezier curve is to the control polygon, the more impossible the intersection between two adjacent curves is.

A set of interpolation points is given by $\mathrm{P}_{\mathrm{i}}$. To obtain the curve through each interpolation point, two auxiliary points $\mathrm{P}_{1}$ and $\mathrm{P}_{\mathrm{n}+1}$ at any location are set. To keep retention type of the curve in the vicinity of two endpoints, they have the same convexity as the two endpoints $\mathrm{P}_{1}, \mathrm{P}_{2}, \mathrm{P}_{3}$ and $\mathrm{P}_{\mathrm{n}-2}, \mathrm{P}_{\mathrm{n}-1}, \mathrm{P}_{\mathrm{n}}$ have. If $\mathrm{P}_{\mathrm{i}}$ is a closed interpolation polygon, a closed interpolation curve is constructed by setting

$$
P_{0}=P_{n}, \mathrm{P}_{n+1}=\mathrm{P}_{1}, \mathrm{P}_{n+2}=\mathrm{P}_{2}
$$

if $P_{i}$ is an open curve, we must set

$$
P_{0}=2 P_{1}-P_{2}, \quad P_{n+1}=2 P_{n}-P_{n-1}
$$

The function of polygon is represented by $S_{i}(u)$ and the basis function of cubic B-spline curve is represented by $\mathrm{P}_{\mathrm{i}}(\mathrm{u})$. A function is defined by

$$
Q_{i}(u)=(1-\alpha) P_{i}(u)+\alpha S_{i}(u)
$$

When $\alpha$ 趋近 $1, \mathrm{Q}_{\mathrm{i}}(\mathrm{u})$ 趋近 $\mathrm{S}_{\mathrm{i}}(\mathrm{u})$. The basis function of cubic B-spline curve is denoted by

$$
P_{i}(u)=\sum_{k=0}^{3} N_{k, 4}(u) p_{i+k-1} \quad u_{i} \leq u \leq u_{i+1}, i=1, \cdots, n-1
$$

The function of polygon is denoted by

$$
S_{i}(u)=\left(1-s_{i}(u)\right) V_{i}+s_{i}(u) V_{i+1} \quad 0 \leq u \leq 1, i=1, \cdots, n-1
$$

Where $\mathrm{V}_{\mathrm{i}}, \mathrm{V}_{\mathrm{i}+1}$ are adjacent points and $\mathrm{S}_{\mathrm{i}}(\mathrm{u})$ is a singular mixed function on the interval $\left[\mathrm{u}_{\mathrm{i}}, \mathrm{u}_{\mathrm{i}+1}\right]$, which is defined as

$$
s_{i}(u)=1-\left(1-\left(u-u_{i}\right)^{3}\right)^{3}
$$

by

$$
\begin{aligned}
& s\left(u_{i}\right)=0, s\left(u_{i+1}\right)=1 \\
& Q_{i}\left(u_{i}, \alpha\right)=p_{i}, Q\left(u_{i+1}, \alpha\right)=p_{i+1}, i=1,2, \cdots, n-1
\end{aligned}
$$

we can get 


$$
\left\{\begin{array}{c}
\alpha V_{i}=p_{i}-(1-\alpha) P_{i}\left(u_{i}\right) \\
\alpha V_{i+1}=p_{i+1}-(1-\alpha) P\left(u_{i+1}\right)
\end{array} \quad i=1,2, \cdots, n-1\right.
$$

that is

$$
\left\{\begin{array}{l}
Q_{i}(u, \alpha)=(1-\alpha) P_{i}(u)+ \\
{\left[1-s_{i}(u)\right]\left[p_{i}-(1-\alpha) P_{i}\left(u_{i}\right)\right]+s_{i}(u)\left[p_{i+1}-(1-\alpha) P_{i}\left(u_{i+1}\right)\right]} \\
u_{i} \leq u \leq u_{i+1}, i=1,2, \cdots, n-1
\end{array}\right.
$$

Thus, the radian of B-spline curve is changed by adjusting the value of $\alpha$, which make two adjacent smooth curves disjoint and is shown in Fig. 6 .

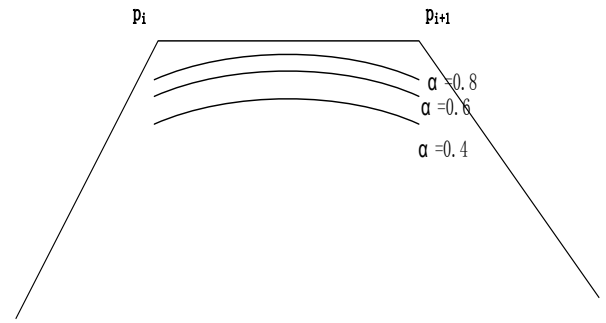

Figure 6. The location of curves and segment

$p_{i} p_{i+1}$ with the change of $\alpha$

As B-spline curve applied in [6], the above method containing mixing factor $\alpha$ takes $\alpha$ as shape parameter, and has the same parameter as the original B-spline curve has. A situation of intersection between two contours can be solved by adjusting the value of $\alpha$.

The Optimization of Contour Annotation. For the contour annotation in [7], the location of annotation should be found. To find relatively flat part in curve, it is necessary to determine three points and calculate the angle between two segments which is generated by connecting two adjacent points. If the angle is larger than 120 degrees, the location is suitable for annotation. However, we cannot find the angle is smaller than 120 degrees, where should the annotation be? Moreover, annotation can be more concentrated when not controlling the location of annotation; the marked texts and numbers can be coincident where contours are dense, which hinder observation and study.

The annotation in this paper consists of two steps. First, for a segment, take the middle point of three sequential adjacent points as the angle point of two segments, calculate the angle $\theta$, firstly consider the location where $\theta$ is the largest and the second largest. Second, for two adjacent curves, annotation of odd contour is added where $\theta$ is the largest while annotation of even contour is added where $\theta$ is the second largest. After the two steps, the interlaced annotation of number and text is achieved, which is clearer as shown in Fig. 7 . 


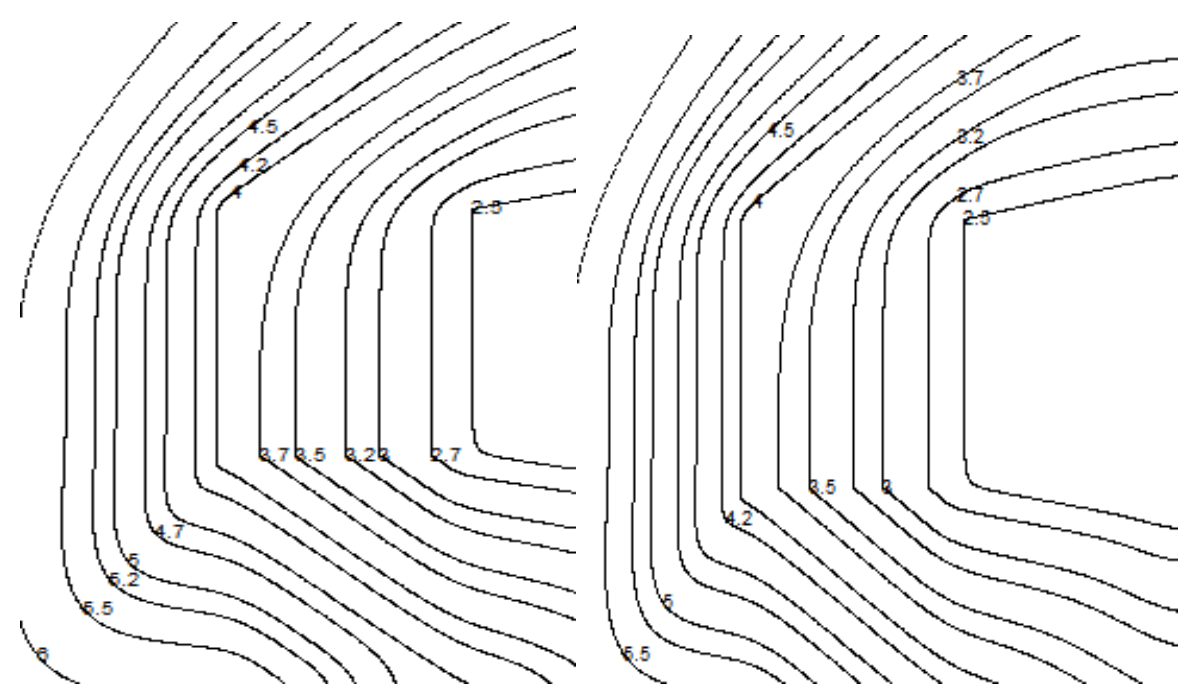

Figure 7. (left) annotation without the above second step, (right) annotation with the above second step

\section{Summary}

This paper combines the Delaunay triangulation, contour tracking, smooth contours generation algorithm, the contours of smooth curves intersect problem have to do further processing to solve the contours intersect problem in the visual. During the experiment, the data: points(coordinates, elevation values, index number), edges(index number, the index number of two points), triangle(index number, the index number of three points) to do a more careful study, avoid tracking process due to the index of triangle edge or index number of points error caused the failure of tracking. And on the basis of others excellent research on the contours marked improvements have been made, making the contour labels is not intensive, facilitate follow-up research staff contours of distribution operations. After doing these actions, we can apply it on the weather forecast [8] graphic display for easy observation of the overall rainfall [9] distribution, can also be used in geological prospecting [10] on the geological distribution can be targeted Research analysis.

Can be found in the process of solving the intersection curve, how to find the case of the intersection curve, there is a problem. In this regard, in the next work will be done a experiment in java environment: for polylines and generate a few curves, control points can be moved freely polyline (based on disjoint polyline), observed changes in the curve, then detected cases generate curves intersect.

\section{References}

[1] J. Yu, L. Pin and C. ZHENG: A Comparative Research on Methods of Delaunay Triangulation [J]. Journal of Image and Graphics (2010), p.1158. (In Chinese)

[2] J. Yu and X. Wu: An improved rectangular grid contour tracing algorithm [J] .Henan Normal University (Natural Science) (2008), p.34. (In Chinese)

[3] C.E. Liu, Z.C. Wang and Y.G. Wang: Comparative analysis contour tracing method [J].Qinghai Science and Technology (2005), p.39. (In Chinese)

[4] Z.B. Wang and R.Z. Peng, Z.G. Gong: B-spline curve generation principle and implementation [J] .Shihezi University (Natural Science)(2009), p.118. (In Chinese)

[5] L.L. HAN, H.S. SHI: Discussing algorithm for label of isograms [J]. Computer Engineering and Design (2006), p.39. (In Chinese)

[6] Leonidas, J.Guibas and J. Stolfi: Primitivies for the manipulation of General Subdivision and the Computation of Voronoi Diagram. ACM Transaction on Graphics (1985), p.74. 
[7] S.X. Li, J.X. Chen and X. Chen: Drawing of the Three Gorges rainfall contour map [J] use irregular triangular mesh. People Yangtze River (2005), p.44. (In Chinese)

[8] X.L. Liu and W.F. Zhao: Automatic meteorological data observing contour drawing system [J] .Meteorology (2009), p.102. (In Chinese)

[9] L.L. Han: Geological formation and draw contour map [D]. Xi'an University of Science and Technology (2006). (In Chinese)

[10]Z.Y. Liu, Z.B. Dong, S.Y. Yin and L.L. Ren: Extreme precipitation on changes and trends in Jinan, Shandong Province [J] Earth Environment (2013), p.1506. (In Chinese) 